\title{
ON COEFFICIENT INEQUALITIES IN THE CLASS $\Sigma$
}

\author{
By Seiji Konakazawa and Mitsuru Ozawa
}

$\S 0$. Introduction. Let $\Sigma$ denote the class of functions

$$
f(z)=z+\sum_{n=1}^{\infty} b_{n} z^{-n}
$$

analytic and univalent in $|z|>1$. In this paper we shall prove the following theorems.

THEOREM 1. (i) If $-1 / 3 \leqq \lambda<1$, then

$$
\operatorname{Re}\left\{b_{5}+b_{1} b_{3}+b_{2}^{2}+\lambda\left(2 b_{3}+b_{1}^{2}\right)\right\} \leqq \frac{1}{3}\left(1+3 \lambda^{2}-\lambda^{3}\right) .
$$

Equality occurs if and only if

$$
f(z)=z\left\{\left(1+3 \lambda z^{-2}+3 b_{2} z^{-3}+3 \lambda z^{-4}+z^{-6}\right)^{2 / 3}+2\left(b_{1}-\lambda\right) z^{-2}\right\}^{1 / 2}
$$

where $b_{2}$ is real and $\left|b_{2}\right| \leqq(1 / 3) \min \left\{2+6 \lambda, 2(1-\lambda)^{3 / 2}\right\}$, with $\arg \left(b_{1}-\lambda\right)=\pi(\bmod .2 \pi)$ and $0 \leqq\left|b_{1}-\lambda\right| \leqq(1 / 2) \min \left\{\left[(2+6 \lambda)+3 b_{2}\right]^{2 / 3},\left[(2+6 \lambda)-3 b_{2}\right]^{2 / 3}\right\}$, or with $\arg \left(b_{1}-\lambda\right)$ $=\pi / 3,-\pi / 3(\bmod .2 \pi)$ and $0 \leqq\left|b_{1}-\lambda\right| \leqq(1 / 2) \min \left\{\left[2(1-\lambda)^{3 / 2}+3 b_{2}\right]^{2 / 3},\left[2(1-\lambda)^{3 / 2}\right.\right.$ $\left.\left.-3 b_{2}\right]^{2 / 3}\right\}$.

(ii) If $1 \leqq \lambda$, then

$$
\operatorname{Re}\left\{b_{5}+b_{1} b_{3}+b_{2}^{2}+\lambda\left(2 b_{3}+b_{1}^{2}\right)\right\} \leqq \lambda .
$$

Equality occurs if and only if

$$
f(z)=z\left\{1+2 b_{1} z^{-2}+z^{-4}\right\}^{1 / 2}
$$

where $b_{1}$ is real and $-1 \leqq b_{1} \leqq 1$.

THEOREM 2.

$$
\operatorname{Re}\left\{b_{4}+b_{1} b_{2}\right\} \leqq 2 / 5 .
$$

Extremal functions must satisfy

$$
f\left(z^{2}\right)^{5 / 2}-\frac{5}{2} b_{1} f\left(z^{2}\right)^{1 / 2}=z^{5}+e^{i \theta} z^{-5} .
$$

Received November 27, 1986 
Duren [2] proved the inequality in Theorem 1 (i) when $\lambda=0$. And Kubota [4] proved the inequality in Theorem 2 when $b_{1}$ is real.

Our proofs depend on Schiffer's variational method [6], and our key lemmas are that the extremal functions omit the value 0 in $|z|>1$, which are proved by making use of Bombieri's theorem [1].

\section{$\S 1$. Proof of Theorem 1 .}

1.1. Some Lemmas. Firstly we assume that $-1 / 3 \leqq \lambda<1$.

LEMMA 1.1. Every extremal function $f(z)$ omits the value 0 in $|z|>1$.

Proof. Applying Schiffer's variational method to this problem, we find the associated quadratic differential

$$
Q(w) d w^{2}=w^{2}\left(w^{2}-2\left(b_{1}-\lambda\right)\right) d w^{2} .
$$

Assume first that $b_{1}-\lambda \neq 0$. Put $b_{1}-\lambda=\left|b_{1}-\lambda\right| e^{\imath \beta}$. It is clear that the trajectories of $Q(w) d w^{2}$ are symmetric with respect to the origin. Let $\Delta$ denote the critical trajectories of $Q(w) d w^{2}$. Assume that $\beta \neq n \pi / 3$. On the ray $J_{1}=$ $\left\{\right.$ ite $\left.e^{i \beta / 2}: 0<t<\infty\right\}$,

$$
\operatorname{Im}\left\{Q\left(i t e^{i \beta / 2}\right)\left(d\left(i t e^{i \beta / 2}\right)\right)^{2}\right\}=-t^{2}\left(t^{2}+2\left|b_{1}-\lambda\right|\right) \sin 3 \beta(d t)^{2} \neq 0 .
$$

Hence by Bombieri's theorem [1] $\bar{J}_{1}$ meets a component of $\Delta$ which goes through the origin only at the origin. The same fact is true for $-J_{1}=\left\{\right.$ ıt $\left.e^{i \beta / 2}:-\infty<t<0\right\}$. On $J_{2}=\left\{t e^{\imath \beta / 2}: 0<t<\left(2\left|b_{1}-\lambda\right|\right)^{1 / 2}\right\}$,

$$
\operatorname{Im}\left\{Q\left(t e^{\imath \beta / 2}\right)\left(d\left(t e^{2 \beta / 2}\right)\right)^{2}\right\}=t^{2}\left(t^{2}-2\left|b_{1}-\lambda\right|\right) \sin 3 \beta(d t)^{2} \neq 0 .
$$

Hence $\bar{J}_{2}$ meets one component of $\Delta$ at most at the origin and another component at most at the critical point $\left(2\left(b_{1}-\lambda\right)\right)^{1 / 2}$. The similar fact holds for $-J_{2}$. On $J_{3}=\left\{t e^{i \beta / 2}:\left(2\left|b_{1}-\lambda\right|\right)^{1 / 2}<t<\infty\right\}$,

$$
\operatorname{Im}\left\{Q\left(t e^{\imath \beta / 2}\right)\left(d\left(t e^{i \beta / 2}\right)\right)^{2}\right\}=t^{2}\left(t^{2}-2\left|b_{1}-\lambda\right|\right) \sin 3 \beta(d t)^{2} \neq 0 .
$$

Hence $\bar{J}_{3}$ meets $\Delta$ at most at the critical point $\left(2\left(b_{1}-\lambda\right)\right)^{1 / 2}$, because three critical trajectories must meet at $\left(2\left(b_{1}-\lambda\right)\right)^{1 / 2}$ with equal angles. The similar fact holds for $-J_{3}$. Furthermore, on the short segments $J_{\alpha}=\left\{t e^{\imath(\beta+\alpha) / 2}: 0<t<\varepsilon\right\}$,

$$
\begin{aligned}
& \operatorname{Im}\left\{Q\left(t e^{i(\beta+\alpha) / 2}\right)\left(d\left(t e^{i(\beta+\alpha) / 2}\right)\right)^{2}\right\} \\
& \quad=t^{2}\left(t^{2} \sin (3 \beta+3 \alpha)-2\left|b_{1}-\lambda\right| \sin (3 \beta+2 \alpha)\right)(d t)^{2} \neq 0
\end{aligned}
$$

for all sufficiently small $\alpha$ and $\varepsilon$. Thus none of the four trajectories which tend to the origin can be tangential along $J_{2}$. The same fact holds along $-J_{2}$. Hence by the fact that the four trajectories meet at the origin with equal 
angles, we know that each of them remains in each of the quadrants divided by $J_{2}+J_{3}, J_{1},\left(-J_{2}\right)+\left(-J_{3}\right)$ and $-J_{1}$.

Assume that $\beta=0$. Then

$$
Q(w) d w^{2}=w^{2}\left(w^{2}-2\left|b_{1}-\lambda\right|\right) d w^{2} .
$$

In this case $\Delta$ has three components and is symmetric with respect to the real axis. In the case of $\beta=2 n \pi / 3$, the shape of $\Delta$ is the rotated one of $\Delta$ in the case of $\beta=0$.

Assume that $\beta=\pi$. Then

$$
Q(w) d w^{2}=w^{2}\left(w^{2}+2\left|b_{1}-\lambda\right|\right) d w^{2} .
$$

In this case $\Delta$ has only one component and is symmetric with respect to the real axis. In the case of $\beta=(2 n+1) \pi / 3$, the shape of $\Delta$ is the rotated one of $\Delta$ in the case of $\beta=\pi$.

Now assume that $b_{1}-\lambda=0$. Then

$$
Q(w) d w^{2}=w^{4} d w^{2} .
$$

In this case $\Delta$ consists of six rays meeting at the origin with equal angles.

We denote the image of $|z|=1$ by the extremal function $f(z)$ by $\Gamma$. $\quad \Gamma$ is on the trajectories of $Q(w) d w^{2}$. So $\Gamma$ must be on $\Delta$ and go through the origin, because the conformal centre

$$
\frac{1}{2 \pi} \int_{0}^{2 \pi} f\left(e^{i \theta}\right) d \theta=0
$$

This completes the proof.

We prepare two more lemmas. The next one is a special case of Jenkins' general coefficient theorem.

LEMmA 1.2. (Jenkins, e.g. [5, Theorem 8.12.]) Let

$$
\phi(w)=w+a_{2} w^{-2}+a_{3} w^{-3}+a_{4} w^{-4}+a_{5} w^{-5}+\cdots
$$

be univalent and admissible for the quadratic differential

Then

$$
Q(w) d w^{2}=\left(A_{0} w^{4}+A_{1} w^{3}+A_{2} w^{2}+A_{3} w\right) d w^{2} .
$$

$$
\operatorname{Re}\left(A_{0} a_{5}+A_{1} a_{4}+A_{2} a_{3}+A_{3} a_{2}+A_{0} a_{2}^{2}\right) \leqq 0 .
$$

If equality holds, then

$$
\frac{Q(\psi(w))}{Q(w)} \phi^{\prime}(w)^{2} \equiv 1 .
$$

LEMMA 1.3. If $-1 / 3 \leqq \lambda \leqq 1$, then 


$$
\operatorname{Re}\left\{b_{5}+b_{1} b_{3}+\lambda\left(2 b_{3}+b_{1}^{2}\right)\right\} \leqq \frac{1}{3}\left(1+3 \lambda^{2}-\lambda^{3}\right)
$$

for all odd functions $f(z)$ in $\Sigma$. Equality occurs if and only if

$$
f(z)=z\left\{\left(1+3 \lambda z^{-2}+3 \lambda z^{-4}+z^{-6}\right)^{2 / 3}+2\left(b_{1}-\lambda\right) z^{-2}\right\}^{1 / 2}
$$

with $\arg \left(b_{1}-\lambda\right)=\pi(\bmod .2 \pi)$ and $0 \leqq\left|b_{1}-\lambda\right| \leqq(1 / 2)(2+6 \lambda)^{2 / 3}$, or with $\arg \left(b_{1}-\lambda\right)$ $=\pi / 3,-\pi / 3(\bmod .2 \pi)$ and $0 \leqq\left|b_{1}-\lambda\right| \leqq 2^{-1 / 3}(1-\lambda)$.

Proof. From any odd function $f(z)=z+b_{1} z^{-1}+b_{3} z^{-3}+b_{5} z^{-5}+\cdots$ in $\Sigma$, we obtain the univalent function

$$
f\left(z^{1 / 2}\right)^{2}=z+c_{0}+c_{1} z^{-1}+c_{2} z^{-2}+\cdots
$$

where $c_{1}=2 b_{3}+b_{1}^{2}$ and $c_{2}=2\left(b_{5}+b_{1} b_{3}\right)$. Hence

$$
b_{5}+b_{1} b_{3}+\lambda\left(2 b_{3}+b_{1}^{2}\right)=\frac{1}{2}\left(c_{2}+2 \lambda c_{1}\right) .
$$

By this relation and Jenkins' results ([3], Lemma 3 and Corollary 10), we obtain the desired result.

1.2. SCHIFFER'S DIFFERENTIAL EQUATION. We denote the extremal function by

Put

$$
f(z)=z+b_{1} z^{-1}+b_{2} z^{-2}+b_{3} z^{-3}+b_{4} z^{-4}+b_{5}+z^{-5}+\cdots .
$$

$$
\phi(w)=-f\left(-f^{-1}(w)\right)=w-2 b_{2} w^{-2}-\left(2 b_{4}+4 b_{1} b_{2}\right) w^{-4}-4 b_{2}^{2} w^{-5}+\cdots .
$$

Because $\Gamma=f(|z|=1)$ is on the critical trajectories of the quadratic differential $Q(w) d w^{2}=w^{2}\left(w^{2}-2\left(b_{1}-\lambda\right)\right) d w^{2}$, we have

$$
\phi(w)^{2}\left(\phi(w)^{2}-2\left(b_{1}-\lambda\right)\right) \psi^{\prime}(w)^{2}=w^{2}\left(w^{2}-2\left(b_{1}-\lambda\right)\right)
$$

by making use of Lemma 1.2. Expanding the left hand side and comparirg the coefficients, we have

$$
b_{4}+b_{1} b_{2}+\lambda b_{2}=0 \text {. }
$$

Hence the extremal function $f(z)$ satisfies Schiffer's differential equation

$$
\begin{gathered}
f(z)^{2}\left(f(z)^{2}-2\left(b_{1}-\lambda\right)\right) z^{2} f^{\prime}(z)^{2} \\
=z^{6}+2 \lambda z^{4}-\left(2 b_{3}+b_{1}^{2}\right) z^{2}-6\left(b_{5}+b_{1} b_{3}+b_{2}^{2}+\frac{2}{3} \lambda\left(2 b_{3}+b_{1}^{2}\right)\right)-\left(2 \bar{b}_{3}+\bar{b}_{1}^{2}\right) z^{-2}+2 \lambda z^{-4}+z^{-6} .
\end{gathered}
$$

We denote the right hand side by $q(z)$. Then

$$
q(z) \leqq 0 \quad \text { on }|z|=1 \text {. }
$$

1.3. Proof of Theorem 1(i). Suppose that $b_{1}-\lambda=0$. Then Schiffer's dif- 
ferential equation (1) becomes

$$
\begin{aligned}
& f(z)^{4} z^{8} f^{\prime}(z)^{2}=z^{6} q(z) \\
& \quad=\left[\prod_{\jmath=1}^{6}\left(z-e^{\imath \alpha_{j}}\right)\right]^{2}=\left[z^{6}+A z^{5}+B z^{4}+C z^{3}+D z^{2}+E z+F\right]^{2},
\end{aligned}
$$

by Lemma 1.1. Comparing coefficients, we have $A=C=E=0, B=\lambda, F^{2}=1$, $D F=\lambda, D=-b_{3}-\lambda^{2}$ and $-3\left(b_{5}+b_{1} b_{3}+b_{2}^{2}+(2 / 3) \lambda\left(2 b_{3}+b_{1}^{2}\right)\right)=F+\lambda D$. It follows that $F=-1, D=-\lambda$ and $b_{3}=\lambda-\lambda^{2}$ by the fact that $q(z) \leqq 0$ on $|z|=1$. Hence we have

$$
b_{5}+b_{1} b_{3}+b_{2}^{2}+\lambda\left(2 b_{3}+b_{1}^{2}\right)=\frac{1}{3}\left(1+3 \lambda^{2}-\lambda^{3}\right) \text {. }
$$

And the extremal function $w=f(z)$ must satisfy

$$
w^{4} z^{8}(d w / d z)^{2}=\left[z^{6}+\lambda z^{4}-\lambda z^{2}-1\right]^{2} .
$$

By solving this differential equation we have

$$
w=z\left(1+3 \lambda z^{-2}+3 b_{2} z^{-3}+3 \lambda z^{-4}+z^{-6}\right)^{1 / 3}
$$

where $b_{2}$ is real and $\left|b_{2}\right| \leqq(1 / 3) \min \left\{2+6 \lambda, 2(1-\lambda)^{3 / 2}\right\}$.

Now assume that $b_{1}-\lambda \neq 0$ and put $b_{1}-\lambda=\left|b_{1}-\lambda\right| e^{2 \beta}$ as before.

Case a). Suppose that $\beta=(2 n+1) \pi / 3$ and that $\Gamma=f(|z|=1)$ contains both of critical points $\pm\left(2\left(b_{1}-\lambda\right)\right)^{1 / 2}$. Then we know as above that

$$
b_{5}+b_{1} b_{3}+b_{2}^{2}+\lambda\left(2 b_{3}+b_{1}^{2}\right)=\frac{1}{3}\left(1+3 \lambda^{2}-\lambda^{3}\right)
$$

and that the extremal function must satisfy

$$
w^{2}\left(w^{2}-2\left(b_{1}-\lambda\right)\right) z^{8}(d w / d z)^{2}=\left[z^{6}+\lambda z^{4}-\lambda z^{2}-1\right]^{2} .
$$

By solving this differential equation we have

$$
w=z\left\{\left(1+3 \lambda z^{-2}+3 b_{2} z^{-3}+3 \lambda z^{-4}+z^{-6}\right)^{2 / 3}+2\left(b_{1}-\lambda\right) z^{-2}\right\}^{1 / 2}
$$

where $b_{2}$ is real and $\left|b_{2}\right| \leqq(1 / 3) \min \left\{2+6 \lambda, 2(1-\lambda)^{3 / 2}\right\}$, with $\arg \left(b_{1}-\lambda\right)=\pi$ (mod. $2 \pi)$ and $0<\left|b_{1}-\lambda\right| \leqq(1 / 2) \min \left\{\left[(2+6 \lambda)+3 b_{2}\right]^{2 / 3},\left[(2+6 \lambda)-3 b_{2}\right]^{2 / 3}\right\}$, or with $\arg \left(b_{1}-\lambda\right)=\pi / 3,-\pi / 3(\bmod .2 \pi)$ and $0<\left|b_{1}-\lambda\right| \leqq(1 / 2) \min \left\{\left[2(1-\lambda)^{3 / 2}+3 b_{2}\right]^{2 / 3}\right.$, $\left.\left[2(1-\lambda)^{3 / 2}-3 b_{2}\right]^{2 / 3}\right\}$.

Case b). Suppose that $\beta=(2 n+1) \pi / 3$ and $\Gamma$ contains exactly one of the critical points $\pm\left(2\left(b_{1}-\lambda\right)\right)^{1 / 2}$. Because $q(-z)=q(z)$ we have

$$
f(-z)^{2}\left(f(-z)^{2}-2\left(b_{1}-\lambda\right)\right) f^{\prime}(-z)^{2}=f(z)^{2}\left(f(z)^{2}-2\left(b_{1}-\lambda\right)\right) f^{\prime}(z)^{2}
$$

in $|z|>1$. If $f\left(z_{0}\right)=\left(2\left(b_{1}-\lambda\right)\right)^{1 / 2}$ for some $z_{0},\left|z_{0}\right|>1$, then

$$
f\left(-z_{0}\right)=0 \text { or } \quad f\left(-z_{0}\right)=-\left(2\left(b_{1}-\lambda\right)\right)^{1 / 2} .
$$


By Lemma 1.1 we have $f\left(-z_{0}\right)=-\left(2\left(b_{1}-\lambda\right)\right)^{1 / 2}$. But this contradicts the assumption. Hence this case cannot occur.

Case c). Now suppose that $\Gamma$ does not contain the critical points $\pm\left(2\left(b_{1}-\lambda\right)\right)^{1 / 2}$. By Lemma 1.1 and the fact that $q(-z)=q(z)$ we can put

$$
\begin{aligned}
f(z)^{2}\left(f(z)^{2}-2\left(b_{1}-\lambda\right)\right) z^{8} f^{\prime}(z)^{2}=z^{6} q(z) \\
=\left[\left(z^{2}-e^{2 \imath \alpha_{1}}\right)\left(z^{2}-e^{2 \imath \alpha_{2}}\right)\right]^{2}\left(z^{2}-r^{2} e^{2 \imath \alpha_{3}}\right)\left(z^{2}-r^{-2} e^{2 \imath \alpha_{3}}\right)
\end{aligned}
$$

for some real $\alpha,(\jmath=1,2,3)$ and $r>1$. Putting $w=f(z)$, we have

$$
\begin{aligned}
& w\left(w^{2}-2\left(b_{1}-\lambda\right)\right)^{1 / 2} d w \\
& \quad=z^{-4}\left(z^{2}-e^{2 \imath \alpha_{1}}\right)\left(z^{2}-e^{2 \imath \alpha_{2}}\right)\left(\left(z^{2}-r^{2} e^{2 \imath \alpha_{3}}\right)\left(z^{2}-r^{-2} e^{2 \imath \alpha_{3}}\right)\right)^{1 / 2} d z .
\end{aligned}
$$

Hence

$$
\begin{aligned}
& \int_{\left(2\left(b_{1}-\lambda\right)\right)^{\lambda / 2}}^{f\left(e^{\left.\imath \alpha_{1}\right)}\right.} w\left(w^{2}-2\left(b_{1}-\lambda\right)\right)^{1 / 2} d w \\
& \quad=\int_{r e^{\imath \alpha_{3}}}^{e \imath \alpha_{1}} z^{-4}\left(z^{2}-e^{2 \imath \alpha_{1}}\right)\left(z^{2}-e^{2 \imath \alpha_{2}}\right)\left(\left(z^{2}-r^{2} e^{2 \imath \alpha_{3}}\right)\left(z^{2}-r^{-2} e^{2 \imath \alpha_{3}}\right)\right)^{1 / 2} d z
\end{aligned}
$$

and

$$
\begin{aligned}
& \int_{-\left(2\left(b_{1}-\lambda\right)\right) 1 / 2}^{f\left(-e \imath \alpha_{1}\right)} w\left(w^{2}-2\left(b_{1}-\lambda\right)\right)^{1 / 2} d w \\
& \quad=\int_{-r e^{2 \alpha_{3}}}^{-e^{\imath \alpha_{1}}} z^{-4}\left(z^{2}-e^{2 \imath \alpha_{1}}\right)\left(z^{2}-e^{2 \imath \alpha_{2}}\right)\left(\left(z^{2}-r^{2} e^{2 \imath \alpha_{3}}\right)\left(z^{2}-r^{-2} e^{2 \imath \alpha_{3}}\right)\right)^{1 / 2} d z .
\end{aligned}
$$

The integrand in the right hand side is a single-valued odd function on the domain $D$, the complement of the slits $\left(\infty,-r e^{2 \alpha_{3}}\right),\left(-e^{2 \alpha_{3}} / r, e^{2 \alpha_{3}} / r\right)$ and $\left(r e^{\imath \alpha_{3}}, \infty\right)$. Taking the integral path $\gamma$ in $D$ from $r e^{2 \alpha_{3}}$ to $e^{\imath \alpha_{1}}$ and $-\gamma$ from $-r e^{2 \alpha_{3}}$ to $-e^{2 \alpha_{1}}$, it follows that

$$
\int_{\left(2\left(b_{1}-\lambda\right)\right)^{1 / 2}}^{f\left(e \imath \alpha_{1}\right)} w\left(w^{2}-2\left(b_{1}-\lambda\right)\right)^{1 / 2} d w=\int_{-\left(2\left(b_{1}-\lambda\right)\right) 1 / 2}^{f\left(-e \imath \alpha_{1}\right)} w\left(w^{2}-2\left(b_{1}-\lambda\right)\right)^{1 / 2} d w .
$$

Thus we have

$$
\frac{1}{3}\left(f\left(e^{\imath \alpha_{1}}\right)^{2}-2\left(b_{1}-\lambda\right)\right)^{3 / 2}=\frac{1}{3}\left(f\left(-e^{\imath \alpha_{1}}\right)^{2}-2\left(b_{1}-\lambda\right)\right)^{3 / 2} .
$$

Hence

$$
f\left(e^{\imath \alpha_{1}}\right)=-f\left(-e^{\imath \alpha_{1}}\right) .
$$

By a similar calculation we also have

$$
f\left(e^{\imath \alpha_{2}}\right)=-f\left(-e^{\imath \alpha_{2}}\right) .
$$

Hence $\Gamma$ is symmetric with respect to the origin. So the extremal function 
$f(z)$ must be an odd function. This case is contained in Lemma 1.3. This completes the proof of Theorem 1 (i).

1.4. Proof of Theorem 1 (ii). It follows from Theorem 1 (i) that

$$
\operatorname{Re}\left(b_{5}+b_{1} b_{3}+b_{2}^{2}+2 b_{3}+b_{1}^{2}\right) \leqq 1 .
$$

By making use of the inequality

$$
\operatorname{Re}\left(2 b_{3}+b_{1}^{2}\right) \leqq 1
$$

which is one of Grunsky's inequalities, we have

$$
\begin{aligned}
& \operatorname{Re}\left\{b_{5}+b_{1} b_{3}+b_{2}^{2}+\lambda\left(2 b_{3}+b_{1}^{2}\right)\right\} \\
& \quad=\operatorname{Re}\left(b_{5}+b_{1} b_{3}+b_{2}^{2}+2 b_{3}+b_{1}^{2}\right)+(\lambda-1) \operatorname{Re}\left(2 b_{3}+b_{1}^{2}\right) \\
& \quad \leqq 1+(\lambda-1)=\lambda
\end{aligned}
$$

for all $\lambda \geqq 1$. Equality occurs only for the functions which satisfy $\operatorname{Re}\left(2 b_{3}+b_{1}^{2}\right)$ $=1$. These are

$$
f(z)=z\left(1+2 b_{1} z^{-2}+z^{-4}\right)^{1 / 2}
$$

where $b_{1}$ is real and $-1 \leqq b_{1} \leqq 1$. In fact these functions satisfy $\operatorname{Re}\left\{b_{5}+b_{1} b_{3}+b_{2}^{2}\right.$ $\left.+\lambda\left(2 b_{3}+b_{1}^{2}\right)\right\}=\lambda$. Hence we obtain the desired result.

\section{$\S 2$. Proof of Theorem 2 .}

2.1. A LEMMA. We start again the following

LEMMA 2.1. Every extremal function $f(z)$ omits the value 0 in $|z|>1$.

Proof. The associated quadratic differential of this problem is

$$
Q(w) d w^{2}=w\left(w^{2}-b_{1}\right) d w^{2} .
$$

Take an extremal function $f(z)=z+b_{1} z^{-1}+b_{2} z^{-2}+b_{3} z^{-3}+b_{4} z^{-4}+\cdots$. We can put $b_{1}=\left|b_{1}\right| e^{2 \alpha}$ with $0 \leqq \alpha<4 \pi / 5$ by rotation. The local structure of critical trajectories of $Q(w) d w^{2}$ around the critical points $0,\left(b_{1}\right)^{1 / 2},-\left(b_{1}\right)^{1 / 2}$ and $\infty$ is well known. Let us denote the critical trajectories of $Q(w) d w^{2}$ by $\Delta$. If $b_{1}=0$ then $\Delta$ consists of five rays joining at the origin with equal angles. We suppose that $b_{1} \neq 0$.

Case a). $\alpha=0 . \Delta$ is symmetric with respect to the real axis. Let $J$ be the imaginary axis $\{$ it $:-\infty<t<\infty\}$. Along $J$

$$
\operatorname{Im}\left\{Q(w) d w^{2}\right\}=t\left(t^{2}+b_{1}\right)(d t)^{2} \neq 0
$$


for $t \neq 0$. Hence by Bombieri's theorem $\bar{J}$ meets the component of $\Delta$ which goes through the origin only at the origin. So we can conclude that $\Gamma=f(|z|=1)$ contains the origin because the conformal centre

$$
\frac{1}{2 \pi} \int_{0}^{2 \pi} f\left(e^{i \theta}\right) d \theta=0 \text {. }
$$

Case b). $\alpha=\pi / 5$. Let $J_{1}$ be $\left\{t e^{\pi \tau / 10}: 0<t<\infty\right\}$. Along $J_{1}$

$$
\operatorname{Im}\left\{Q(w) d w^{2}\right\}=t\left(t^{2}-\left|b_{1}\right|\right)(d t)^{2} \neq 0
$$

for $t \neq\left|b_{1}\right|^{1 / 2}$. Hence $\bar{J}_{1}$ meets one component of $\Delta$ at most at the origin and the other component at most at $\left(b_{1}\right)^{1 / 2}$. The similar fact holds along $-J_{1}$. Let $J_{2}$ be $\left\{\right.$ ite $\left.e^{\pi \imath / 10}: 0<t<\infty\right\}$. Along $J_{2}$

$$
Q(w) d w^{2}=-t\left(t^{2}+\left|b_{1}\right|\right)(d t)^{2} .
$$

Hence $J_{2}$ is an orthogonal trajectory and $-J_{2}$ is a critical trajectory. Thus $\Gamma$ passes through the origin by

$$
\frac{1}{2 \pi} \int_{0}^{2 \pi} f\left(e^{i \theta}\right) d \theta=0 .
$$

Case c). $0<\alpha<\pi / 5$ or $\pi / 5<\alpha<2 \pi / 5$. Let $J_{1}$ be $\left\{t e^{2 \alpha / 2}: 0<t<\infty\right\}$. Along $J_{1}$

$$
\operatorname{Im}\left\{Q(w) d w^{2}\right\}=t\left(t^{2}-\left|b_{1}\right|\right) \sin (5 \alpha / 2)(d t)^{2} \neq 0
$$

for $t \neq\left|b_{1}\right|^{1 / 2}$. Hence $\bar{J}_{1}$ meets two components of $\Delta$ at most at the origin and at $\left(b_{1}\right)^{1 / 2}$ respectively. The similar fact holds along $-J_{1}$. On $J_{2}=$ $\left\{t e^{-\pi \imath / 5}: 0<t<\infty\right\}$,

$$
\operatorname{Im}\left\{Q(w) d w^{2}\right\}=t\left|b_{1}\right| \sin (\alpha+2 \pi / 5)(d t)^{2} \neq 0 .
$$

Hence $\bar{J}_{2}$ meets the component of $\Delta$ which passes through the origin only at the origin. The same is true for $-J_{2}$. The similar considerations can be applyed to the lines $\left\{t e^{\pi \imath / 5}:-\infty<t<\infty\right\}$ and $\{i t:-\infty<t<\infty\}$. Now we can readily prove that $\Gamma$ goes through the origin by the above facts, the local structure of critical trajectories of $Q(w) d w^{2}$ around the critical points and

$$
\frac{1}{2 \pi} \int_{0}^{2 \pi} f\left(e^{i \theta}\right) d \theta=0
$$

Case d). $2 \pi / 5 \leqq \alpha<4 \pi / 5$. By the rotation $w=e^{-4 \pi \imath / 5} \zeta, \quad w\left(w^{2}-b_{1}\right) d w^{2}=$ $\zeta\left(\zeta^{2}-b_{1} e^{-2 \pi i / 5}\right) d \zeta^{2}$. It means that this case is essentially included in the above three cases. This completes the proof.

2.2. Proof of Theorem 2. Let $f(z)=z+b_{1} z^{-1}+b_{2} z^{-2}+b_{3} z^{-3}+b_{4} z^{-4}+\cdots$ be an extremal function. We can take its square-root transformation $g(z)=f\left(z^{2}\right)^{1 / 2}$ by Lemma 2.1. Let $F_{5}(w)$ be the fifth Faber polynomial of $g(z)$ defined by 


$$
F_{5}(g(z))=z^{5}+\sum_{n=1}^{\infty} a_{5 n} z^{-n} .
$$

Then Grunsky's inequality says that

$$
\sum_{n=1}^{\infty} n\left|a=a_{5 n}\right|^{2} \leqq 5
$$

and especially

$$
\left|a_{55}\right| \leqq 1
$$

Because $a_{55}=(5 / 2)\left(b_{4}+b_{1} b_{2}\right)$ in this case, we obtain

$$
\left|b_{4}+b_{1} b_{2}\right| \leqq 2 / 5 \text {. }
$$

If equality holds then $\left|a_{55}\right|=1$ and therefore $a_{5 n}=0$ for $n \neq 5$. Hence we have $F_{5}(g(z))=z^{5}+e^{i \theta} z^{-5}$. Since $F_{5}(w)=w^{5}-(5 / 2) b_{1} w$ in this case, we obtain the desired relation

$$
f\left(z^{2}\right)^{5 / 2}-\frac{5}{2} b_{1} f\left(z^{2}\right)^{1 / 2}=z^{5}+e^{i \theta} z^{-5} .
$$

Expanding the left hand side of this relation, we have $b_{2}=0$. It means that $b_{2}=0$ for each extremal function. Thus we can deduce that $\left|b_{4}+b_{1} b_{2}\right|<2 / 5$ if $b_{2} \neq 0$. Moreover, it follows directly from Theorem 2 that if $b_{2}=0$ then $\left|b_{4}\right| \leqq 2 / 5$.

\section{REFERENCES}

[1] Bombieri, E., A geometric approach to some coefficient inequalities for univalent functions, Ann. Scoula Norm. Sup. Pisa 22 (1968), 377-397.

[2] Duren, P.L., Applications of the Garabedian-Schiffer inequality, J. Analyse Math. 30 (1976), 141-149.

[3] Jenkins, J.A., On certain coefficients of univalent functions, Analytic Functions, Princeton Univ. Press (1960), 159-194.

[4] Kubota, Y., On the fourth coefficient of meromorphic univalent functions, Kōdai Math. Sem. Rep. 26 (1975), 267-288.

[5] Pommerenke, Ch., Univalent Functions (with a chapter on quadratic differentials by G. Jensen), Vandenhoeck \& Ruprecht, Göttingen, 1975.

[6] Schiffer, M., A method of variation within the family of simple functions, Proc. London Math. Soc. 44 (1938), 432-449.

Department of Mathematics SCIENCE UNIVERsity OF TOKyo

Noda, ChibA, JAPAN 